\title{
La robótica y el diseño de APP: una posibilidad para potenciar el pensamiento matemático de los estudiantes de pregrado ${ }^{1}$
}

\section{Robotics and APP design: a possibility to enhance the mathematical thinking of undergraduate students}

\author{
E. Murcia y J. C. Henao \\ Recibido: noviembre 21 de 2018 - Aceptado: enero 15 de 2020
}

\begin{abstract}
Resumen- En el contexto educativo nacional y para los estudiantes de los niveles de educación básica y media, sus apropiaciones de los conceptos matemáticos tienden a ser bajos y esto se hace especialmente evidente cuando ingresan a la de educación superior. Es necesario implementar metodologías activas e innovadoras que promuevan en el estudiante nuevas dinámicas para el desarrollo de un Saber matemático; para dar una respuesta a este problema, desde el grupo de investigación Entre Ciencia e Ingeniería de la Facultad de Ciencias Básica e Ingeniería de la Universidad Católica de Pereira, se propone llevar a cabo una propuesta metodológica basada en el uso de dispositivos robóticos LEGO MINDSTORMS y plataformas móviles como App Inventor con el propósito de que el estudiante mejore sus niveles de competencia en los pensamientos numérico, variacional y geométrico.
\end{abstract}

Palabras clave- Dispositivos robóticos, App Inventor, Pensamiento variacional, Pensamiento numérico, Lego Mindstorms.

Abstract-In the national educational context and for students at the basic and secondary education levels, their appropriations of mathematical concepts tend to be low and this becomes especially evident when they enter higher education. It is necessary to implement active and innovative methodologies that promote in the student new dynamics for the development of

\footnotetext{
${ }^{1}$ Este artículo es uno de los resultados del proyecto denominado Implementación de didáctica apoyada en Robots Lego Mind Storm y Diseño de metodologías apoyadas en App Inventor para potenciar el pensamiento matemático en los estudiantes que cursan a los diferentes programas de matemáticas de la UCP
}

E. Murcia, Universidad Católica de Pereira, Pereira Colombia, email: euclides.murcia@ucp.edu.co.

J. C. Henao, Universidad Católica de Pereira, Pereira Colombia, email: juan.henao@ucp.edu.co

Como citar este artículo: Murcia, E. y Henao, J. C. La robótica y el diseño de app: una posibilidad para potenciar el pensamiento matemático de los estudiantes de pregrado, Entre Ciencia e Ingeniería, vol. 14, no. 27, pp. 35-40, enero-junio año. DOI: https://doi.org/10.31908/19098367.1749.

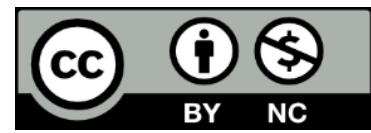

Attribution-NonCommercial 4.0 Intenational (CC By-NC 4.0) a mathematical Knowledge; To answer to this problem, from the research group Entre Ciencia e Ingeniería of the Faculty of Basic Sciences and Engineering of the Católica de Pereira University, it is proposed to carry out a methodological proposal based on the use of robotic devices LEGO MINDSTORMS and mobile platforms such as App Inventor with the purpose that the student improves their levels of competence in numerical, variational and geometric thoughts.

Keywords-Robotic devices, App inventor, Variational thinking, numerical thinking, Lego Mindstorms.

\section{INTRODUCCIÓN}

$\square^{n}$ n cuanto a la importancia que presenta la apropiación de los conceptos matemáticos por parte de los estudiantes que ingresan a los diferentes programas de la Universidad Católica de Pereira, se propone desde el grupo de investigación Entre Ciencia e Ingeniería una serie de metodologías de innovación pedagógica con el propósito de que los docentes que orientan asignaturas puedan implementar estas estrategias apoyadas en Tecnologías de la Información y de la Comunicación que promuevan en el estudiante el interés por potenciar el pensamiento matemático fundamentado en tres categorías fundamentales: pensamiento variacional, pensamiento numérico y pensamiento geométrico; este artículo presenta los resultados de la implementación del uso de plataformas móviles y dispositivos robóticos LEGO MINDSTORMS con estudiantes de los programas de Ingenierías, Administración de Empresas, Negocios Internacionales y Psicología.

Por otro lado, la investigación que se desarrolló sigue las pautas de bajo un enfoque cuantitativo, mediante la implementación de un diseño experimental como se plantea en [1], para ello se elige inicialmente un solo grupo experimental y a su vez este mismo se erige también como grupo control, además dentro de este diseño investigativo se escoge un modelo de carácter cuasi experimental para datos apareados con un solo grupo. Finalmente se plantea una prueba de hipótesis de diferencia de medias con el propósito de verificar la pertinencia de esta metodología en estudiantes que cursan la asignatura de matemáticas en un grupo de 24 estudiantes. 


\section{ANTECEDENTES}

La matemática como proceso de pensamiento, ha estado estrechamente ligada a la evolución misma de la humanidad en razón a que se precisa de herramientas cognitivas que facilite comprender la realidad para eventualmente predecirla, controlarla y modificarla; nociones como el conteo y las mismas representaciones geométricas son el resultado de construcciones colectivas en diferentes culturas y momentos de la historia.

El Ministerio de Educación Nacional en Colombia propone una serie de Lineamientos Curriculares, Estándares Básicos de Competencia, y Derechos Básicos de Aprendizaje en muchas áreas del conocimiento incluyendo las matemáticas, que son documentos de referencia en la construcción de los proyectos educativos institucionales en los colegios [2]-[4]; que propenden por una reducción de las brechas sociales pero también por mejorar la competitividad del país. Sobre estos constructos es que se desarrollan nuevas propuestas didácticas de trasformación que eventualmente lleva a resignificar los programas académicos de las instituciones de educación superior.

Para la construcción adecuada y consistente del pensamiento numérico en los estudiantes, las acciones deben favorecer entre otras cosas, que el alumno: 1) Reconozca los diferentes tipos de sistemas de numeración que existen. 2) Comprenda la composición de los diferentes conjuntos numéricos 3) Interiorice la estructura de las operaciones básicas en los conjuntos numéricos. 4) Entienda las propiedades de los conjuntos numéricos, y para ello es de gran importancia el dominio conceptual que pueda tener el estudiante en procesos lógico-matemáticos como "medir", "contar" y "ordenar". El mismo Ministerio de Educación Nacional en la serie de Lineamientos para la Enseñanza de las matemáticas, insiste en la correcta construcción del concepto de los números naturales, los enteros, los racionales, que se asocian a los subprocesos de conteo y medición.

Con base en lo anterior, se puede evidenciar que tanto el proceso de conteo como el de medición hacen parte de los objetos del saber que deben ser adquiridos correctamente y así mismo lo contempla Chevallard cuando en [5] se refiere a las diferentes trasformaciones adaptativas que presenta un contenido de saber, el cual termina convirtiéndose en un objeto de conocimiento. Se debe mencionar, que esta estructura de pensamiento que lleva el estudiante se convierte en la base para apropiar conceptos más complejos como los de análisis discreto y continuo entre otros.

Así mismo, es necesario establecer un sentido lógico al lenguaje matemático para que este adquiera significado para el estudiante, esto conlleva a establecer que todo cálculo matemático que realice, debe reflejar una buena precisión numérica y una estructura algorítmica secuencial, que den sustento a lo que se quiere representar o modelar; así, se debe insistir en el desarrollo de las ideas matemáticas y el sistema de signos con lo cual el estudiante progresaría de manera directa en el desarrollo de elementos semióticos y esto a portas de interpretar de una mejor manera los sistemas de signos matemáticos [6].

Al mismo tiempo las TIC son un elemento fundamental de la mediación entre saber-estudiante-maestro como lo expresa
[7], se podría establecer desde allí una relación que implante una sinergia entre pensamiento matemático y el uso de las Tecnologías de la Información y de la Comunicación con el fin de generar nuevos objetos de conocimiento fundamentados en un ambiente tecnológico que apoye de alguna manera la concreción del pensamiento matemático [8].

La implementación de las TIC en el aula de clase, demanda que se lleven a cabo metodologías propias de la disciplina, en asocio con una propuesta pedagógica consistente en el marco de lo definido en [9], y que implique entre otras cosas, la resolución de problemas usando algoritmos que trasciendan a la simple memorización o repetición sin sentido de instrucciones [10], se podría precisar que con todo esto que el estudiante puede realizar juicios de los hallazgos encontrados en los cálculos matemáticos que realiza y así poder tomar decisiones del proceso que se encuentra desarrollando, no obstante este tipo de acciones le bridan la habilidad para comunicar, procesar e interpretar información numérica.

De otro lado, cuando se habla del pensamiento espacial en analogía con los pensamientos numérico y variacional, se debe precisar que los currículos deben de estar adaptados a que el estudiante pueda transformar su realidad contextual y poder transfigurar estos espacios y sus relaciones en objetos del saber propio de este tipo de pensamiento con lo cual este implementará la habilidad de medir, que en última instancia se convierte en una de las nociones más importantes que un estudiante matemáticamente competente debe adquirir como se promueve de manera explícita en [11] donde se aborda el problema de las Inteligencias Múltiples de Gardner.

\section{INTERVENCIÓN Y RESULTADOS INICIALES}

Teniendo como base el desarrollo del pensamiento numérico del estudiante apoyado en pensamientos como el geométrico, el variacional y el de medida, es necesario que él reconozca la estructura y propiedades del conjunto de los números reales, ver Fig. 1.

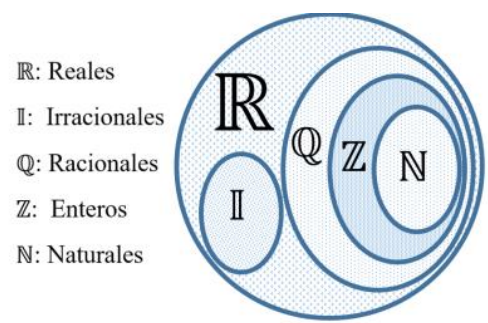

Fig. 1. Conjunto de los números reales

Así entonces, el hecho de que el estudiante pueda reconocer el conjunto de los números reales, implica hacer el tránsito por los enteros -quienes fundamentan la teoría discreta-, siguiendo con los números racionales, para llegar a los irracionales -que sustentan conceptos como el de la continuidad y medición- y finalizar con la conformación de los números reales; la adecuada estructuración cognitiva consolida en el estudiante un saber mínimo que potencia su estructuras explicativas dado un contexto matemático, con lo cual podrá resolver eficientemente problemas.

Por otro lado, la estructura numérica ya mencionada converge en los siguientes conjuntos. 
- $\mathbb{N}:\{1,2,3, \ldots, \ldots \infty\}$

(Números naturales).

- $\mathbb{Z}:\{-\infty, \ldots, \quad-100, \ldots \quad 1, \quad 2,3, \ldots, \ldots \quad \infty\}$

(Números enteros).

- $\mathbb{Q}:\left\{-\infty, \ldots,-\frac{3}{4}, \ldots, \frac{2}{5}, \ldots, \frac{5}{2} \ldots \infty\right\}$

(Números racionales de la forma $\mathrm{p} / \mathrm{q}$, donde $q \neq 0$.)

- II: $\{-\infty, \ldots,-\sqrt{2}, \ldots, \sqrt{3} \ldots, \pi \ldots \infty\}$

(Números irracionales).

A su vez, se requiere que el estudiante pueda identificar la forma como se fueron constituyendo los diferentes conjuntos numéricos, lo cual conllevaría a que este pueda plantear una estructura mental del uso de estos cuando lo requiera, dando paso a lo que se denomina objeto de saber y así el estudiante pueda llevar a cabo operaciones matemáticas básicas (suma, diferencia, multiplicación y división), reconociendo las propiedades básicas y siendo protagonista y artífice de su aprendizaje [12] y después deberá desarrollar actividades que requieran de cálculo mental para resolver problemas que demanden de este tipo de dinámicas.

Se debe agregar que en el momento en el que el estudiante identifica correctamente los conjuntos numéricos a los que pertenece una cantidad dada y que al mismo tiempo resuelva sin inconvenientes actividades que contengan operaciones básicas (sin el uso de calculadoras), se procede a llevar a cabo, la interposición de otras operaciones como la potenciación, la radicación y la logaritmación con todas sus propiedades [13]. Por otro lado, existe una relación muy estrecha entre el pensamiento numérico y el pensamiento variacional, por ende, teniendo en cuenta las propuestas de [2] cuando se refiere a que el estudiante debe detectar los criterios que determinan ciertas regularidades dentro de los conjuntos numéricos ya mencionados y a su vez identificar patrones que se repiten de manera frecuente los cuales se hacen evidentes en secuencias o sucesiones que dan vida a otra temática de importancia como las progresiones.

Se realiza una serie de intervenciones se llevó a cabo con un grupo de 24 estudiantes de la formación en Ingeniería usando una metodología basada en el uso de dispositivos robóticos Lego Mindstorms, además de efectuar actividades en la plataforma App Inventor. Esta intervención inicia con un diagnóstico de los niveles de competencia de matemáticas que presentan los estudiantes a través de un Pretest, el cual arrojó los resultados que se muestran en la Tabla I.

TABLA I.

NOTAS DE PRE-TEST

\begin{tabular}{|c|c|c|c|c|c|}
\hline E1 & E2 & E3 & E4 & E5 & E6 \\
\hline 2,7 & 1,3 & 1,2 & 2,6 & 1,3 & 3,2 \\
\hline E7 & E8 & E9 & E10 & E11 & E12 \\
\hline 1,3 & 1,4 & 1,3 & 0,8 & 1,3 & 2,2 \\
\hline E13 & E14 & E15 & E16 & E17 & E18 \\
\hline 1,3 & 1,3 & 3,3 & 1,3 & 2,2 & 1,8 \\
\hline E19 & E20 & $\mathbf{E 2 1}$ & $\mathbf{E 2 2}$ & $\mathbf{E 2 3}$ & $\mathbf{E 2 4}$ \\
\hline 2,5 & 1,4 & 4 & 2,5 & 2 & 1,8 \\
\hline
\end{tabular}

La media, la varianza y desviación estándar de las notas que se obtuvieron se muestran en la tabla II.

TABLA II.

MEDIA, VARIANZA Y DESVIACIÓN ESTÁNDAR (PRE-TEST)

\begin{tabular}{|l|c|}
\hline Categoría & Valor \\
\hline Media & 1,9 \\
\hline Varianza & 0,66 \\
\hline Desviación Estándar & 0,81 \\
\hline
\end{tabular}

Teniendo en cuenta esta información, la cual evidencia que los estudiantes presentan un nivel bajo de competencia en estas dos áreas del saber matemático (pensamiento variacional y numérico), se procede a implementar una propuesta didáctica con robots Lego Mindstorms y plataformas App Inventor; algunos de los ejercicios de aplicación en el aula, se presentan a continuación.

La primera actividad fue que los estudiantes dibujen sobre un papel bond o sobre un pliego de cartulina un plano polar, semejante al mostrado en la Fig. 2; posteriormente, los estudiantes deben proponer un programa o una rutina desde la interfaz para computadora, de modo que el robot:

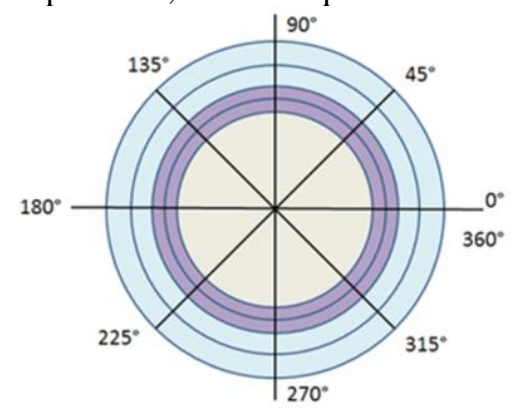

Fig. 2. Plano porlar

a. Gire a la izquierda $135^{\circ}$

b. Gire a la derecha $225^{\circ}$

c. Gire a la izquierda $90^{\circ}$

d. Gire a la derecha $45^{\circ}$

e. Dé tres vueltas hacia la derecha.

f. Dé cuatro vueltas hacia la izquierda.

Realizada esta actividad en plataforma LabView que es la interfaz de programación de los robots Lego Mindstorms, o bien sea realizando la programación en los bloques computacionales que presenta este dispositivo (Fig. 3), el estudiante adquiere conceptos y categorías como medida angular y posición relativa.

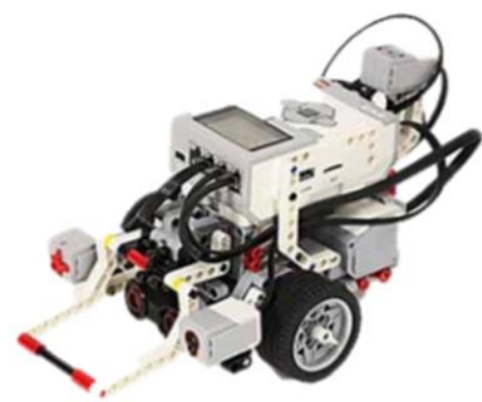

Fig 3. Dispositivo Robótico LEGO MINDSTORMS

Una segunda actividad consiste en hacer mover el robot de diferentes maneras, pero con la condición de que espere cierto 
tiempo entre dos acciones diferentes. Para ello, debe construir una rutina en la interfaz para $\mathrm{PC}$, de modo que el robot.

a. Avance siete segundos, luego espere por ocho segundos más en reposo y posteriormente vuelva a su posición inicial.

b. Recorra el perímetro de un rectángulo de cualquier dimensión, pero en cada vértice espere 8 segundos.

En este ejercicio, el estudiante debe tener en cuenta que, al realizar la programación del dispositivo robótico, bien sea desde la consola propia del robot, o desde la plataforma LabView con la que cuenta el dispositivo, es necesario que use parámetros de tiempo y prever que el robot se mueva en alguna dirección, para ello se le propone como reto, hacer mover el dispositivo siguiendo un rectángulo. Con este tipo de prácticas, el estudiante aprende conceptos geométricos y variacional aplicados a la resolución de problemas contextualizados.

En relación con el uso de la plataforma App Inventor, a los estudiantes se les plantean actividades donde debían desarrollar aplicaciones móviles para la resolución de un problema matemático; como ejemplo se exponen dos casos particulares, el primero una aplicación que soluciona una ecuación cuadrática, el segundo una aplicación que calcula la combinatoria de un valor dado, ver Fig. 4, este tipo de ejercicios constituyen lo que se llama un Entorno Personal de Aprendizaje (PLE) [14], se debe precisar que el desarrollo de este tipo de elementos por su propósito educativo y por su estructura podría visualizarse como un Objeto Virtual de Aprendizaje (OVA), tal como lo manifiesta en [15].

Estas dos actividades establecen de manifiesto que el estudiante debe poner a prueba su pensamiento lógico en primera instancia para poder desarrollar la aplicación, y también debe aplicar los criterios y conceptos del pensamiento numérico y variacional que exigen estas mismas. Es necesario recalcar que fomentar en el estudiante estos tipos de pensamientos matemáticos conlleva a que este pueda apropiar de manera casi implícita pensamientos como el de sistema de datos, métrico y el estadístico [16].
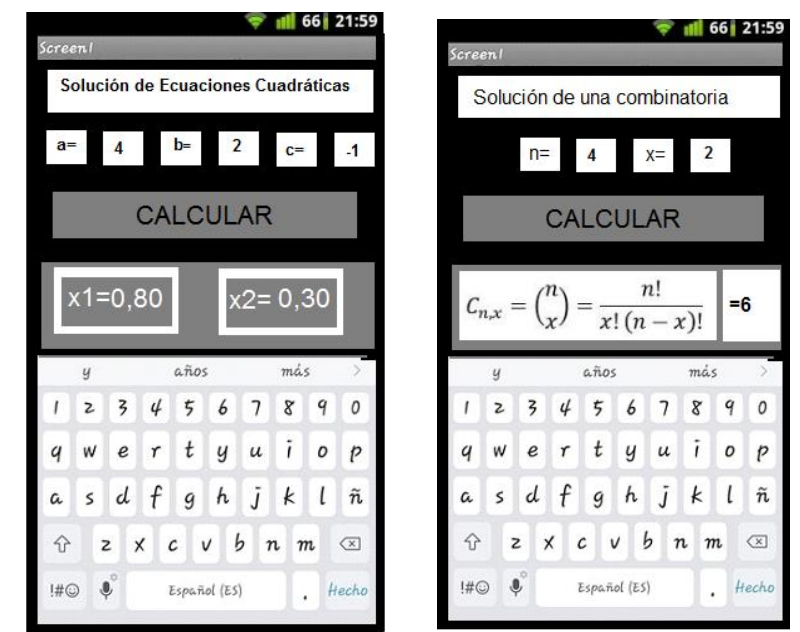

Fig. 4. Ejemplo de un programa en App Inventor

\section{DISCUSIÓN Y RESULTADOS}

\section{A. Resultados del Post-Test}

Posteriormente a la intervención, se aplican de nuevo pruebas estandarizadas a los 24 estudiantes cuyos resultados se muestran en las Tabla III y Tabla IV.

TABLA III

NotAs DE POST-TEST

\begin{tabular}{|c|c|c|c|c|c|}
\hline E1 & E2 & E3 & E4 & E5 & E6 \\
\hline 4,8 & 3,3 & 3,1 & 4,8 & 3,1 & 4,8 \\
\hline E7 & E8 & E9 & E10 & E11 & E12 \\
\hline 3,0 & 4,0 & 4,8 & 0,0 & 4,1 & 3,8 \\
\hline E13 & E14 & E15 & E16 & E17 & E18 \\
\hline 4,8 & 3,1 & 4,1 & 4,8 & 4,0 & 4,0 \\
\hline E19 & E20 & $\mathbf{E 2 1}$ & $\mathbf{E 2 2}$ & $\mathbf{E 2 3}$ & $\mathbf{E 2 4}$ \\
\hline 4,0 & 4,1 & 4,8 & 3,8 & 3,1 & 4,1 \\
\hline
\end{tabular}

TABLA IV

MEDIA, VARIANZA Y DESVIACIÓN ESTÁNDAR (POST-TEST)

\begin{tabular}{|l|c|}
\hline Categoría & Valor \\
\hline Media & 3,8 \\
\hline Varianza & 1,08 \\
\hline Desviación Estándar & 1,04 \\
\hline
\end{tabular}

Tal como se muestra en las tablas anteriores los resultados obtenidos por los estudiantes son aparentemente mejores comparados con los obtenidos en la prueba del Pre-Test, el promedio pasa de 1,9 a 3,8; siendo este un resultado satisfactorio, lo que induce la conveniencia de implementar la metodología.

\section{B. Prueba de hipótesis.}

Por lo que se refiere el enfoque investigativo de carácter cuantitativo apoyado mediante un diseño experimental, se plantea una prueba de hipótesis para datos apareados con una sola muestra de 24 estudiantes, tal y como se describe a continuación en el planteamiento o formulación de la hipótesis Estadística.

Но: $\boldsymbol{\mu} \mathbf{2}-\boldsymbol{\mu} \mathbf{1}=0$

Ho: la metodología tradicional entrega resultados iguales que la metodología implementada mediante Dispositivos Robóticos y App Inventor

Ha: los estudiantes presentan un promedio Ha: $\boldsymbol{\mu} \mathbf{2}-\boldsymbol{\mu} \mathbf{1}>0$ de notas mayor mediante la implementación de la metodología usando Dispositivos Robóticos y App Inventor

Dado que se cumple la hipótesis del investigador, se puede afirmar que existe una relación directa entre los aprendizajes alcanzados cuando se usa estos recursos tecnológicos.

\section{Puntajes obtenidos}

Los puntajes que obtuvieron los estudiantes tanto en el PreTest como en el Post-Test se muestran en la Tabla V. 
TABLA V.

PUNTAJES Y DIFERENCIAS ENTRE EL PRE-TEST Y POST-TEST

\begin{tabular}{|l|c|c|c|c|c|c|c|c|}
\hline ESTUDIANTE & E1 & E2 & E3 & E4 & E5 & E6 & E7 & E8 \\
\hline PRE TEST & 2,7 & 1,3 & 1,2 & 2,6 & 1,3 & 3,2 & 1,3 & 1,4 \\
\hline POST TEST & 4,8 & 3,3 & 3,1 & 4,8 & 3,1 & 4,8 & 3,0 & 4,0 \\
\hline DIFERENCIAS & 2,1 & 2,0 & 1,9 & 2,2 & 1,8 & 1,6 & 1,7 & 2,6 \\
\hline
\end{tabular}

\begin{tabular}{|l|c|c|c|c|c|c|c|c|}
\hline ESTUDIANTE & E9 & E10 & E11 & E12 & E13 & E14 & E15 & E16 \\
\hline PRE TEST & 1,3 & 0,8 & 1,3 & 2,2 & 1,3 & 1,3 & 3,3 & 1,3 \\
\hline POST TEST & 4,8 & 0,0 & 4,1 & 3,8 & 4,8 & 3,1 & 4,1 & 4,8 \\
\hline DIFERENCIAS & 3,5 & 0,8 & 2,8 & 1,6 & 3,5 & 1,8 & 0,8 & 3,5 \\
\hline
\end{tabular}

\begin{tabular}{|l|c|c|c|c|c|c|c|c|}
\hline ESTUDIANTE & E17 & E18 & E19 & E20 & E21 & E22 & E23 & E24 \\
\hline PRE TEST & 2,2 & 1,8 & 2,5 & 1,4 & 4,0 & 2,5 & 2,0 & 1,8 \\
\hline POST TEST & 4,0 & 4,0 & 4,0 & 4,1 & 4,8 & 3,8 & 3,1 & 4,1 \\
\hline DIFERENCIAS & 1,8 & 2,2 & 1,5 & 2,7 & 0,8 & 1,3 & 1,1 & 2,3 \\
\hline
\end{tabular}

$\bar{d}=2,0$ Media de las diferencias

$s_{d}=0,8$ Desviación de las diferencias

$n=24$ Número de estudiantes

$\alpha=0.05$ Nivel de significancia

$v=23$ Grados de libertad (n-1)

$\mathrm{t} 1=12,2$ Estadístico de prueba

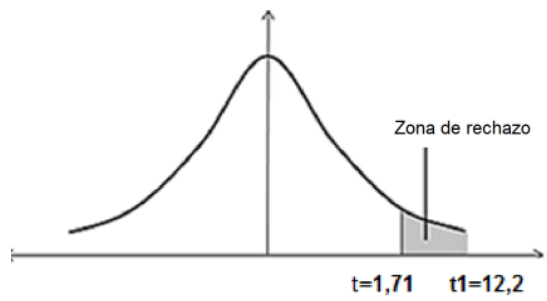

Fig. 5. Estadístico de Prueba

Partiendo del planteamiento de la hipótesis como se menciona anteriormente, se puede evidenciar que con un nivel de significancia de 0,05 y 23 grados de libertad, el resultado del estadístico de prueba $(\mathrm{t} 1=12,12)$, se ubica en la región de rechazo, tal como se muestra en la Fig. 5 (lo cual rechaza la hipótesis nula) por lo tanto se puede concluir con una significancia de 0,05 que la metodología mediada por Dispositivos Robóticos y App Inventor para potenciar el pensamiento matemático de los estudiantes; entrega mejores resultados que una metodología tradicional.

\section{CONSIDERACIONES ADICIONALES}

Con respecto a que uno de los propósitos de las matemáticas es que el ser humano pueda adquirir herramientas de pensamiento para resolver de manera eficiente cualquier tipo de problema que se le presente en su cotidianidad, para ello Polya, establece dentro de su teoría de solución de problemas matemáticos algunos pasos como: 1) Interpretar el problema. 2) Desarrollar un plan de resolución. 3) Ejecutar dicho plan. 4) Revisar y contrastar resultados para determinar la veracidad y confiabilidad de los resultados obtenidos [17]. A todo esto, se suma la importancia de que el estudiante tenga un conocimiento del conjunto numérico donde está desarrollando las operaciones con criterios matemáticos y la forma de abordar un algoritmo lógico para poder obtener resultados consistentes.
Adicional a estos procesos de desarrollo de pensamiento matemático, el estudiante debe adquirir también habilidades de comprensión lectora [18], con el propósito de que él pueda ejecutar el primer paso planteado por Polya en la solución de problemas, el cual hace referencia a la interpretación del problema, seguidamente el estudiante debe realizar un esquema inicial de solución, para lo cual necesita de usar cada una de las herramientas matemáticas adquiridas en las primeras fases de la educación primaria y consolidadas en la educación secundaria. En estos primeros pasos es donde se recomienda estimular a los estudiantes para que se acerquen a las matemáticas usando como base la geometría para luego consolidar con pensamientos como el métrico y el numérico la habilidad de medir y contar, lo que fortalece a su vez elementos que son fundamentales en los cursos de cálculo diferencial. De la misma manera la apropiación del pensamiento numérico conlleva a que el estudiante adapte habilidades como las de recolección y organización de la información fundamentando de manera directa objetos del saber de la estadística descriptiva

Por otra parte, y acorde a la evolución tecnológica que se ha venido presentando en las últimas décadas, cabe destacar la estrecha relación entre el pensamiento matemático, el pensamiento lógico y el desarrollo de algoritmos que promueven desarrollar en el estudiante un pensamiento concreto el cual inicia con un procedimiento abstracto lo cual termina con un contenido real y tangible que el estudiante puede verificar constante mente (conocimiento matemático perdurable) tal como lo expone [7].

\section{CONCLUSIONES}

En matemáticas es comúnmente aceptado que hay tres procesos importantes en el desarrollo del pensamiento; estos son medir, contar y ordenar, que sustentan a su vez procesos más complejos; brindar al estudiante recursos tecnológicos tanto digitales como no digitales, reducen las brechas educativas que se dan en los sistemas de educación, y que se hacen más visibles cuando se comparan los sectores público y privado.

Los resultados obtenidos en el diagnóstico o pre-test, permiten validar la afirmación que existen importantes vacíos conceptuales que generan dificultades académicas cuando ingresan los estudiantes de los niveles de educación media a la Universidad Impactando negativamente los índices de calidad, permanencia y promoción y así mismo, la utilización de metodologías y didácticas asociadas a las tecnologías de la información y la comunicación, especialmente cuando éstas son de naturaleza digital, tienen un mayor nivel de aceptación por parte de los estudiantes [19] y esto implica que los procesos de enseñanza se ven simplificados en razón a un mayor nivel de motivación con permanencia dentro del sistema educativo de los alumnos [20].

Finalmente, tanto la implementación de una metodología pedagógica apoyada en dispositivos robóticos que fomente la creación de aplicaciones para dispositivos móviles mediante App Inventor permite potenciar el pensamiento lógico de los estudiantes debido a que en ambas propuestas se requiere que el estudiante siga una secuencia de pasos y desarrolle 
algoritmos para llegar a la solución de un problema de esta índole.

\section{AGRADECIMIENTOS}

Los autores quieren manifestar su agradecimiento a la Universidad Católica de Pereira y muy especialmente a la Facultad de Ciencias Básicas e Ingeniería por el apoyo prestado en el desarrollo de la propuesta de investigación

\section{REFERENCIAS}

[1] R. Hernandez-Sampieri, C. Fernandez-Collado, and P. Baptista Lucio, Metodologia de la Investigación, 6th ed., vol. 1, no. 1. Mexico, Mexico: McGraw-Hill, 2014.

[2] Ministerio de Educación Nacional, Estándares Básicos de Competencia en Lenguaje, Matemáticas, Ciencias y Ciudadanas. Bogotá: Ministerio de Educación Nacional, 2006.

[3] Ministerio de Educación Nacional, "Lineamientos Curriculares en el área de Matemáticas," Ministerio de Educación Nacional, Bogotá, Colombia, 1998.

[4] Ministerio de Educación Nacional, "Matríz de referencia en matemáticas," Bogotá, Colombia, 2016.

[5] Y. Chevallard, La transposición didáctica. Del Saber Sabio al Saber Enseñado. AIQUE, 1999.

[6] B. D'Amore and M. I. Fandiño Pinilla, "Reflexiones teóricas sobre las bases del enfoque ontosemiótico de la Didáctica de la Matemática," Actas del Segundo Congr. Int. Virtual sobre el Enfoque Ontosemiótico del Conoc. y la Instr. Matemáticos, no. 2007, pp. 1-17, 2017.

[7] H. E. Bosch, M. S. Bergero, C. A. Nasso, M. M. Perez, and M. C. Rampazzi, "Innovaciones didácticas para ciencias y matemática asistidas por TIC," Rev. Iberoam. Educ. en Tecnol. y Tecnol. en Educ., vol. 1 , no. 19 , pp. 60-64, 2017.

[8] E. Murcia Londoño and H. Córdoba, "Uso de las Tics y objetos de aprendizaje para la enseñanza de las matemáticas en la UCPR," Entre Cienc. e Ing., vol. 6, no. 6, pp. 129-149, 2006.

[9] J. C. Henao López and E. Murcia Londoño, "Educación matemática en Colombia, una perspectiva evolucionaria," Entre Cienc. e Ing., vol. 9, no. 18, pp. 23-30, 2015.

[10] M. D. P. Díaz López, N. D. M. Torres López, and M. del C. Lozano Segura, "Nuevo Enfoque En La Enseñanza De Las Matemáticas, El Método ABN," Int. J. Dev. Educ. Psychol., vol. 3, no. 1, p. 431, 2017.

[11] L. E. Díaz Posada, S.-P. Varela Londoño, and L. P. Rodríguez Burgos, "Inteligencias múltiples e implementación del currículo: avances, tendencias y oportunidades," Rev. Psicodidáctica, vol. 22, no. 1, pp. 69-83, 2017.

[12] P. Ruiz Bernardo, L. Sánchez Tarazaga, and R. Mateu Pérez, "La innovación pedagógica de la mano de la investigación--- acción para mejorar la calidad de las prácticas externas de los Grados de Maestro/a en Educación Primaria y Educación Infantil," Rev. Electrónica Interuniv. Form. del Profr., vol. 21, no. 1, pp. 33-49, 2018.

[13] O. Aya Corredor, M. I. Cano Villamil, and D. C. García Caro, "Algunas consideraciones didácticas sobre el concepto de logaritmo y de función logarítmica y sus posibilidades en la educación básica," Universidad Pedagógica Nacional, 2016.

[14] J. A. Garcia Martínez and M. Gonzalez Sanmamed, "Cómo generan y gestionan contenidos los estudiantes de educación de Costa Rica: una contribución al estudio de su entorno personal de aprendizaje," Digit. Educ. Rev., vol. 36, no. 1, pp. 15-34, 2019.

[15] E. Murcia Londoño and H. Córdoba Vargas, "Enseñar matemáticas usando objetos virtuales de aprendizaje en la Universidad Católica de Pereira," Entre Cienc. e Ing., vol. 5, no. 10, pp. 148-168, 2011.

[16] A. Gorina Sánchez and I. Alonso Berenguer, "La formación de Pensamiento Estadístico en el nivel Universitario," Órbita Pedagógica, vol. 12, no. 1, pp. 41-54, 2014.

[17] M. L. Meneses Espinal and D. Y. Peñalosa Gelvez, "Método de Pólya como estrategia pedagógica para fortalecer la competencia resolución de problemas matemáticos con operaciones básicas," Zo. Próxima, no. 31, pp. 8-25, 2019.

[18] M. Y. Canales Alfaro, "Comprensión lectora y resolución de problemas matemáticos en estudiantes de un colegio privado de Lima," Rev.
Investig. en Psicol., vol. 21, no. 2, p. 215, 2019.

[19] L. E. Peláez Valencia and B. E. Osorio Patiño, "Medición del nivel de aprendizaje con dos escenarios de formación: uno tradicional y otro con TIC," Entre Cienc. e Ing., vol. 18, no. 1, pp. 59-66, 2015.

[20] E. Murcia Londoño, J. L. Arias Vargas, and S. M. Osorio Montoya, "Software educativo para el buen uso de las TIC," Entre Cienc. e Ing., vol. 19, no. 1, pp. 114-125, 2016.

Juan Carlos Henao López. Nació en Pereira, Colombia el 14 de enero de 1977 y estudió su pregrado en Ingeniería Eléctrica en la Universidad Tecnológica de Pereira y una maestría en educación con la Universidad de Santander, actualmente realiza estudios de Doctorado en Ciencias de la Educación en la Universidad de Cuauhtémoc (México). Ejerce profesionalmente como docente para la Secretaria de Educación de Pereira, la Universidad de Caldas y la Universidad Católica de Pereira donde actualmente se encuentra vinculado laboralmente y pertenece al grupo de investigación Entre ciencia e Ingeniería. Entre sus campos de interés está la didáctica, la robótica, las TIC.

ORCID: https://orcid.org/0000-0002-9988-7371

Euclides Murcia Londoño. Nació en Bogotá, Colombia el 1 de noviembre de 1975 y estudió su pregrado en Matemáticas y Computación en la Universidad del Quindío y una maestría en Enseñanza de las Matemáticas con la Universidad Tecnológica de Pereira, actualmente realiza estudios de Doctorado en Ciencias de la Educación en la Universidad de Cuauhtémoc (México). Ejerce profesionalmente como docente para la Secretaria de Educación de Pereira y la Universidad Católica de Pereira donde actualmente se encuentra vinculado laboralmente y pertenece al grupo de investigación Entre ciencia e Ingeniería. Entre sus campos de interés está la didáctica, la robótica, las TIC y la Estadística.

ORCID: https://orcid.org/0000-0001-9069-519X 